\title{
A Localization Algorithm for Underwater Wireless Sensor Networks Based on Ranging Correction and Inertial Coordination
}

\author{
Ying Guo $^{1,2^{*}}$, Xiaoyue Kang ${ }^{1}$, Qinghe Han ${ }^{1}$ and Jingjing Wang ${ }^{1}$ \\ ${ }^{1}$ Qingdao University of Science and Technology \\ Shandong, Qingdao 266061 - China \\ ${ }^{2}$ Arizona State University \\ Arizona, Tempe 85287 - American \\ [e-mail: guoying@qust.edu.cn] \\ *Corresponding author: Ying Guo
}

Received November 19, 2018; revised March 12, 2019; accepted April 18, 2019; published October 31, 2019

\begin{abstract}
Node localization is the basic task of underwater wireless sensor networks (UWSNs). Most of the existing underwater localization methods rely on ranging accuracy. Due to the special environment conditions in the ocean, beacon nodes are difficult to deploy accurately. The narrow bandwidth and high delay of the underwater acoustic communication channel lead to large errors. In order to reduce the ranging error and improve the positioning accuracy, we propose a localization algorithm based on ranging correction and inertial coordination. The algorithm can be divided into two parts, Range Correction based Localization algorithm (RCL) and Inertial Coordination based Localization algorithm (ICL). RCL uses the geometric relationship between the node positions to correct the ranging error and obtain the exact node position. However, when the unknown node deviates from the deployment area with the movement of the water flow, it cannot communicate with enough beacon nodes in a certain period of time. In this case, the node uses ICL algorithm to combine position data with motion information of neighbor nodes to update its position. The simulation results show that the proposed algorithm greatly improves the positioning accuracy of unknown nodes compared with the existing localization methods.
\end{abstract}

Keywords: wireless sensor networks, underwater, node location, ranging correction, inertial coordination

The work was supported by Natural Science Foundation of Shandong Province (ZR2016FQ10), National Natural Science Foundation of China (No. 61671261), Key Research and Development Program of Shandong Province (No. 2016GGX101007). 


\section{Introduction}

Recently, underwater wireless sensor networks (UWSNs) have attracted much attention and provided sufficient technical support to many application fields, such as ocean environment monitoring, natural disaster prevention and military defense [1-2]. Localization technology is one of the key technologies of UWSNs [3-6]. However, due to the complex underwater environment, the ground node location method cannot be directly applied to UWSNs [7], UWSNs should use different underwater localization methods.

UWSNs localization mainly faces the following difficulties: (1) In UWSNs, the underwater acoustic channel has special characteristics such as narrow bandwidth, high propagation delay, and severe multipath scattering [8-9], which lead to large ranging error and greatly affect the accuracy of the ranging-based localization algorithm. (2) Because the deployment of underwater nodes is large, and the number of beacon nodes is small and the distribution is sparse, the localization algorithm relying on high-density beacon nodes cannot be used. (3) More importantly, all underwater nodes constantly moving by the influence of ocean currents and tides. The localization algorithm must consider the moving characteristics of the nodes [10-11]. These ubiquities in the ocean environment make positioning underwater nodes a challenging task.

Existing underwater sensor networks localization algorithms, such as the multilateral positioning algorithm [12-13]. It is centered on the beacon node, and collects multiple circles with a communication distance as a radius to obtain the coordinates of the node. Two-Phase Time Synchronization-Free Localization Algorithm (TP-TSFLA) [14] uses the geometric relationship between nodes to locate. The Sensor Localization Algorithms (SLA) [15] uses iterative methods to extend the intersection area to the surrounding area to calculate the coordinates of the unknown node. Although these positioning algorithms are theoretically feasible, there are still large positioning errors in practice. The Global Node Selection (GNS) algorithm [16-17] is a typical algorithm for inertial navigation and nodes location update. The strategy of the algorithm is to assume that the node knows the information of the entire network, but due to the mobility of the UWSN nodes and limited storage capacity, this strategy cannot solve the UWSN underwater target tracking problem. There are also a lot of researches on the underwater target tracking problem of UWSN in the literature [18-19], but these results are based on raw measurements, but considering the limited energy and communication bandwidth of UWSN, the original measurement not applicable to UWSN.

In view of the above problems, we analyze the error source of UWSNs in detail. For the range based localization algorithm, the most important error source is the ranging error. At present, most of the existing underwater localization algorithms do not take this into account. They limited to the error caused by nodes movement. In this paper, we design a Range Correction based Localization algorithm (RCL). The geometric relationship between the node positions is used to reduce the ranging error, and the exact position of the node is obtained. This algorithm can correct most of the ranging errors and improve the localization accuracy. For the case that sparse deployment leads to insufficient beacon nodes, we design an Inertial Coordination based Localization algorithm (ICL) based on the movement law of underwater nodes under the influence of ocean currents. The position of the node is obtained based on the inertial navigation position data combined with the motion information of the neighbor nodes. And the simulation results show that the designed algorithm is superior to other existing underwater sensor networks localization algorithms in accuracy and practicability. 
The remaining portion of this paper is organized as follows: Section 2 summarizes the related works. In Section 3, we introduce the network model and proposed the range correction localization algorithm and inertial cooperative localization algorithm. Simulation results are reported in Section 4. We conclude our work in Section 5 with a discussion of future research works.

\section{Related Work}

Underwater node localization technology is one of the most critical technologies in underwater sensor network applications. Without node location information, the collected data has no meaning [20-21]. However, the localization of underwater sensor nodes is affected by many factors such as underwater environment and underwater acoustic communication which makes existing WSN localization algorithms can not be applied to underwater [22-25].

Existing sensor network location schemes could be divided into two groups [26]: range-based and range-free. Rang-based methods need to obtain the distance between the unknown node and the adjacent known node [27]. Typical techniques mainly include Time of Arrival (TOA) [28], Time Difference of Arrival (TDOA) [29], Angle of Arrival (AOA) [30], and Received Signal Strength Indicator (RSSI) [31]. For example, the Underwater Positioning Scheme (UPS) algorithm [32] uses TDOA technology for localization. The algorithm does not need time synchronization, but nodes outside the coverage of four nodes cannot be located, which reduces the localization coverage of the UPS. The Dive and Rise Localization (DNRL) algorithm [33] uses the one-way TOA method for ranging, which enables "quiet" localization, but requires precise time synchronization between nodes. Multi-stage Localization (MSL) [34] is an improvement to the DNRL scheme. The ranging is also a one-way TOA method and the mobility of underwater nodes takes into account, but it also requires precise time synchronization. The AUV-aided Localization (AAL) algorithm [35] is an underwater autonomous aircraft assisted localization scheme. It uses two-way TOA to measure distance, does not require time synchronization, but the communication overhead is large and the localization accuracy is limited by Autonomous Underwater Vehicle (AUV) ranging accuracy. The There-Dimensional Underwater Localization (DUL) [36] algorithm uses two-way TOA ranging, which does not require time synchronization, but the communication delay of this scheme is longer. As time goes by, the ranging error accumulates, which affects the localization accuracy of algorithm.

Rang-free methods do not need to measure the distance between nodes, but the distance of the unknown node is calculated by the connection degree between the unknown node and the surrounding nodes. For example, the Localization with Directional Beacons (LDB) algorithm [37] uses AUV to locate underwater nodes, which is mainly used in static hydrological environments. The communication overhead is small but the accuracy mainly depends on the signal transmission frequency of AUV. The Scalible Localization with Mobility Prediction (SLMP) algorithm [38] can be applied to a dynamic hydrological environment. Both the anchor node and the unknown node estimate the position according to their own motion pattern and previous coordinates [39], but the performance of the algorithm is affected by the localization period and the motion prediction model. Compared with the ranging-based localization algorithms, the rang-free localization algorithms have lower localization accuracy.

Below we use the form of a table to compare the performance of some commonly used underwater wireless sensor network localization algorithms. As shown in Table 1. 
Table 1. Localization algorithm comparison

\begin{tabular}{|c|c|c|c|c|c|c|}
\hline $\begin{array}{c}\text { Algorithm } \\
\text { name }\end{array}$ & $\begin{array}{c}\text { Distributed/ } \\
\text { centralized }\end{array}$ & $\begin{array}{c}\text { Based on } \\
\text { estimation/ } \\
\text { predicting }\end{array}$ & $\begin{array}{c}\text { Anchor } \\
\text { type }\end{array}$ & $\begin{array}{c}\text { Ranging } \\
\text { method }\end{array}$ & $\begin{array}{c}\text { Communica- } \\
\text { tion mode }\end{array}$ & $\begin{array}{c}\text { Time } \\
\text { synchro- } \\
\text { nization }\end{array}$ \\
\hline \hline UPS[32] & distributed & $\begin{array}{c}\text { Based on } \\
\text { estimation }\end{array}$ & $\begin{array}{c}\text { Static } \\
\text { anchor } \\
\text { node }\end{array}$ & $\begin{array}{c}\text { TDOA } \\
\text { ranging }\end{array}$ & silence & No need \\
\hline DNRL[33] & distributed & $\begin{array}{c}\text { Based on } \\
\text { estimation }\end{array}$ & $\begin{array}{c}\text { Mobile } \\
\text { anchor } \\
\text { node }\end{array}$ & $\begin{array}{c}\text { One-way } \\
\text { TOA } \\
\text { ranging }\end{array}$ & silence & need \\
\hline MSL[34] & distributed & $\begin{array}{c}\text { Based on } \\
\text { estimation }\end{array}$ & $\begin{array}{c}\text { Mobile } \\
\text { anchor } \\
\text { node }\end{array}$ & $\begin{array}{c}\text { One-way } \\
\text { TOA } \\
\text { ranging }\end{array}$ & Iteration & need \\
\hline LDB[37] & distributed & $\begin{array}{c}\text { Based on } \\
\text { estimation } \\
\text { estimation }\end{array}$ & $\begin{array}{c}\text { AUV } \\
\text { AOAV } \\
\text { Tanging }\end{array}$ & $\begin{array}{c}\text { No need } \\
\text { to } \\
\text { measure } \\
\text { distance }\end{array}$ & silence & No need \\
\hline SLMP[38] & distributed & $\begin{array}{c}\text { Based on } \\
\text { predicting }\end{array}$ & $\begin{array}{c}\text { Buoy node, } \\
\text { anchor } \\
\text { node, } \\
\text { reference } \\
\text { node }\end{array}$ & $\begin{array}{c}\text { One-way } \\
\text { TOA } \\
\text { ranging }\end{array}$ & Iteration & need \\
& & & Based on & & \\
\hline
\end{tabular}

As discussed above, rang-based localization algorithms has higher accuracy than rang-free localization algorithms, but ranging error will lead to localization error, and the nodes outside the beacon node coverage cannot be located. In addition, such algorithms have higher requirements on the node performance. In this paper, by studying the characteristics of underwater sensor networks and considering the various influencing factors in the underwater node localization process, RCL and ICL algorithm is proposed. Compared with the existing algorithms, the algorithm uses the geometric relationship between nodes to reduce the ranging error and improve the positioning accuracy. In the case that the node deviates from the beacon coverage area, we locate the unknown node in combination with the moving state of the neighbor node. The ranging correction and inertial cooperation mode are different from the existing localization algorithm and have high practical value.

\section{Algorithm design}

In this section, we propose a localization algorithm for underwater wireless sensor networks based on ranging correction, and propose an inertial cooperative positioning mechanism in the case of insufficient beacons.

\subsection{RCL Algorithm}

Underwater acoustic communication greatly influenced by the underwater pressure, temperature salinity and so on that leads to large ranging error, which is the main error source of localization algorithms. In order to improve the localization accuracy, we adopt the 
underwater sensor network localization algorithm based on ranging correction, which mainly includes two steps: The first step is the pre-localization based on the simple multilateral localization algorithm to get the rough position of the node. The second step is the precise localization based on the ranging correction, using the geometric relationship between the nodes to reduce the ranging error and calculate the exact position of the node.

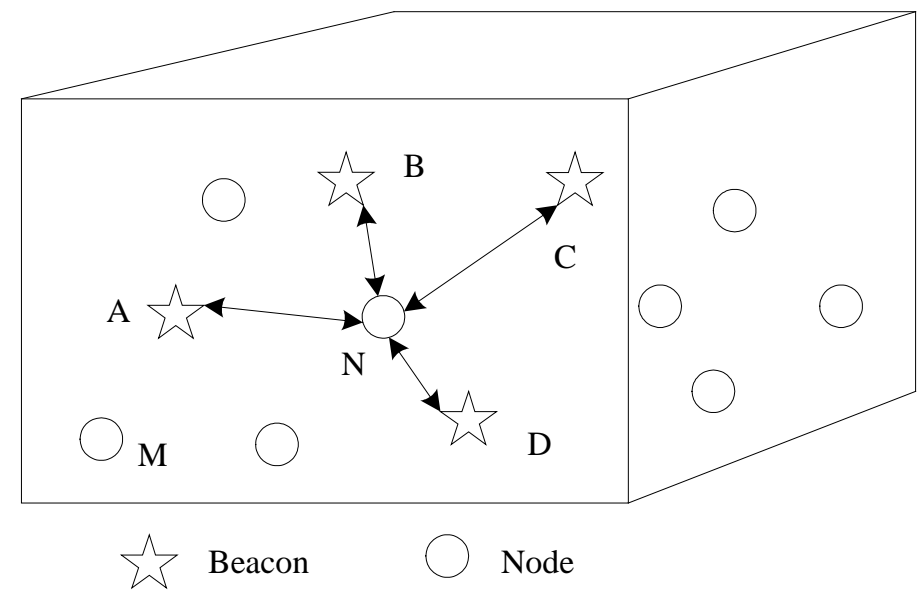

Fig. 1. Pre-localization process

As shown in Fig. 1, the positions of beacons A, B, C, and D are known, and other nodes need to be localized by beacons. In the pre-localization phase, node $\mathrm{N}$ emits a localization request, and beacons $\mathrm{A}, \mathrm{B}, \mathrm{C}$, and $\mathrm{D}$ receive the localization request, and send their coordinates $\left(x_{A}, y_{A}, z_{A}\right),\left(x_{B}, y_{B}, z_{B}\right),\left(x_{C}, y_{C}, z_{C}\right)$ and $\left(x_{D}, y_{D}, z_{D}\right)$ to node $\mathrm{N}$. Node $\mathrm{N}$ uses existing ranging algorithms (such as TOA, TDOA, etc.) to calculate the distance between it and beacons A, B, C, and D, denoted as $d_{A}, d_{B}, d_{C}, d_{D}$, and then uses the principle of multilateral localization algorithm:

$$
q X=g
$$

Among them,

$$
\begin{aligned}
q & =\left[\begin{array}{lll}
2\left(x_{A}-x_{D}\right) & 2\left(y_{A}-y_{D}\right) & 2\left(z_{A}-z_{D}\right) \\
2\left(x_{B}-x_{D}\right) & 2\left(y_{B}-y_{D}\right) & 2\left(z_{B}-z_{D}\right) \\
2\left(x_{C}-x_{D}\right) & 2\left(y_{C}-y_{D}\right) & 2\left(z_{C}-z_{D}\right)
\end{array}\right] \\
g & =\left[\begin{array}{ll}
x_{A}^{2}-x_{D}^{2}+y_{A}^{2}-y_{D}^{2}+z_{A}^{2}-z_{D}^{2}+d_{D}^{2}-d_{A}^{2} \\
x_{B}^{2}-x_{D}^{2}+y_{B}^{2}-y_{D}^{2}+z_{B}^{2}-z_{D}^{2}+d_{D}^{2}-d_{B}^{2} \\
x_{C}^{2}-x_{D}^{2}+y_{C}^{2}-y_{D}^{2}+z_{B}^{2}-z_{D}^{2}+d_{D}^{2}-d_{C}^{2}
\end{array}\right] \\
X & =\left[\begin{array}{l}
x_{N 1} \\
y_{N 1} \\
z_{N 1}
\end{array}\right]
\end{aligned}
$$

The coarse coordinate $\mathrm{N} 1\left(x_{N 1}, y_{N 1}, z_{N 1}\right)$ of node $\mathrm{N}$ can be calculated by Eq. (1).

There are many factors affecting the localization accuracy, and the ranging error is the biggest influencing factor. This is due to the complex and variable underwater environment, 
the underwater sound velocity has a vertical distribution characteristic, which changes with the depth, salinity, temperature and other factors, resulting in a large ranging error [40]. In order to make the localization results more accurate, we propose a precise localization algorithm based on the ranging correction, which is to correct the result of the pre-localization and obtain a more accurate node position.

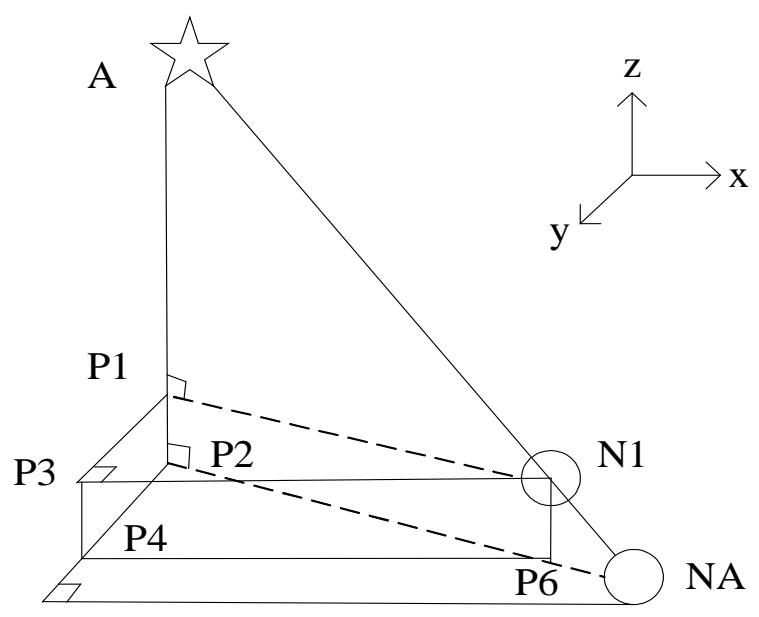

$\mathrm{P} 5$

Fig. 2. Ranging correction diagram

Usually, underwater nodes are equipped with a pressure sensor to obtain their z-axis exact coordinates. Taking beacon $\mathrm{A}$ as an example, the ranging error causes node position $\mathrm{NA}\left(x_{N A}, y_{N A}, z_{N A}\right)$ with accurate z-axis coordinates to be located on the line (or extension) of ANA, and the computed position is N1, as shown in Fig. 2. Make a vertical line AP1 from the position of beacon A to the plane of xy axis. Then make a vertical line from the calculated node position N1 to AP1, the foot point is P1. And then, the node position NA with the exact $\mathrm{z}$-axis coordinates makes a vertical line to AP2, the foot point is P2. The $\triangle \mathrm{AP} 1 \mathrm{~N} 1$ and $\triangle \mathrm{AP} 2 \mathrm{NA}$ are similar triangles, we could get

$$
\frac{A P 1}{A P 2}=\frac{A N 1}{A N A}=\frac{P 1 N 1}{P 2 N A}
$$

Similarly, making a vertical line N1P3 from N1 to the plane of the yz axis, the foot point is P3, making a vertical line NAP5 from NA to the plane of the yz axis, the foot point is P5, making a vertical line P3P4 from P3 to the plane of the xy axis, the foot point is P3, and making a vertical line N1P6 from N1 to the plane of the xy axis, the foot point is P6. $\triangle \mathrm{P} 2 \mathrm{P} 4 \mathrm{P} 6$ and $\triangle \mathrm{P} 2 \mathrm{P} 5 \mathrm{NA}$ are similar triangles, we could get

$$
\frac{P 2 P 4}{P 2 P 5}=\frac{P 4 P 6}{P 5 N A}=\frac{P 2 P 6}{P 2 N A}
$$

Since $P 1 N 1=P 2 P 6$, substitute it to Eq. (3),

$$
\frac{P 2 P 4}{P 2 P 5}=\frac{P 4 P 6}{P 5 N A}=\frac{P 1 N 1}{P 2 N A}
$$

From solve Eqs (2) and (4), we could get 
which is,

$$
\frac{A P 1}{A P 2}=\frac{P 2 P 4}{P 2 P 5}=\frac{P 4 P 6}{P 5 N A}
$$

$$
\frac{z_{N 1}-z_{A}}{z_{N A}-z_{A}}=\frac{y_{N 1}-y_{A}}{y_{N A}-y_{A}}=\frac{x_{N 1}-x_{A}}{x_{N A}-x_{A}}
$$

The coordinate $\left(x_{N A}, y_{N A}\right)$ of position NA could be solved from Eq. (6), and the z-axis coordinate has been measured by the pressure sensor, so the coordinate of NA is $\left(x_{N A}, y_{N A}, z_{N A}\right)$.

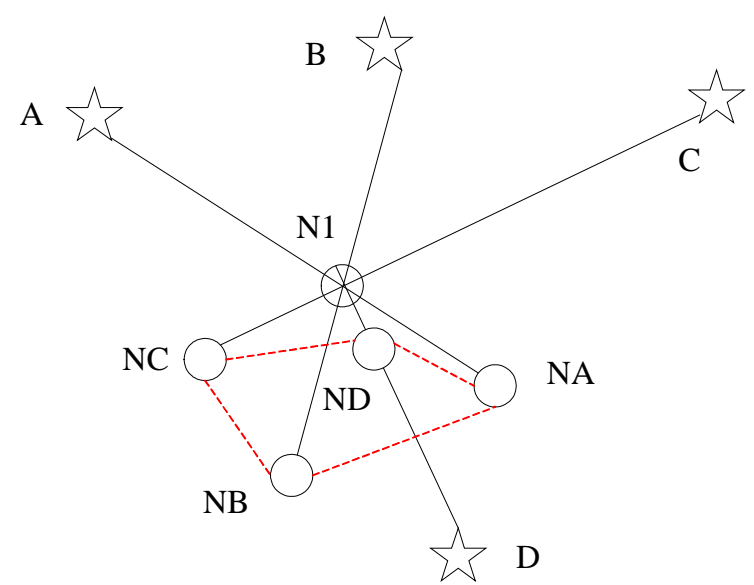

Fig. 3. Ranging correction localization

Repeating the above process for the beacons B, C, and D, the coordinates of NB, NC, and ND can be obtained, recorded as $\left(x_{N B}, y_{N B}, z_{N B}\right),\left(x_{N C}, y_{N C}, z_{N C}\right)$ and $\left(x_{N D}, y_{N D}, z_{N D}\right)$, as shown in Fig. 3. Next, the exact position $\left(x_{N}, y_{N}, z_{N}\right)$ of the node $\mathrm{N}$ is calculated. Where $z_{N}=z_{N A}=z_{N B}=z_{N C}=z_{N D}$, the $x y$ axis coordinates of node $\mathrm{N}$ can be obtained by the centroid algorithm.

$$
\left\{\begin{array}{l}
x_{N}=\frac{x_{N A}+x_{N B}+x_{N C}+x_{N D}}{4} \\
y_{N}=\frac{y_{N A}+y_{N B}+y_{N C}+y_{N D}}{4}
\end{array}\right.
$$

According to above method, the nodes in the network could be located one by one. When the number of beacons is insufficient, the nodes that have completed localization can serve as beacons to assist localization calculation.

\subsection{ICL Algorithm}

Underwater nodes are inevitably moved under the influence of currents and tides. Therefore, the localization algorithm has to re-run at regular intervals to update the node positions.

During the movement of the node, it may deviate from the deployment area in a certain period of time, resulting in failure to communicate with enough beacons. At this time, we adopt a cooperative localization method, in which the inertial navigation position data is 
combined with the motion information of neighbor nodes to obtain the node position.

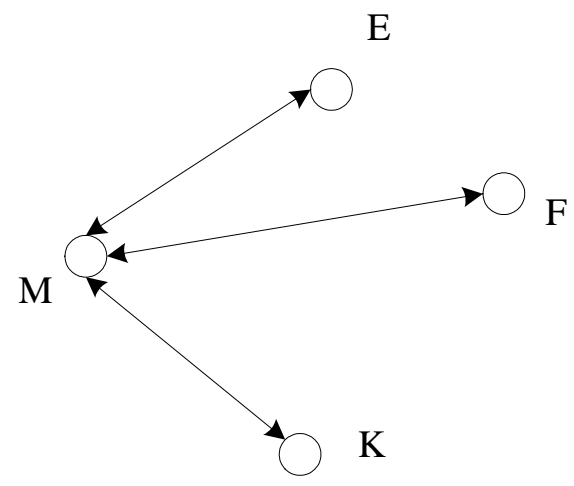

Fig. 4. Inertial cooperative localization diagram

As shown in Fig. 4, the node $\mathrm{M}$ sends a localization request, and only obtains replies from the neighbor nodes E, F, and $\mathrm{K}$. As the number of replies is less than the minimum number of beacons required for calculation (at least 4 beacons are required for 3D underwater localization), it enters the inertial cooperative localization mode. The node $\mathrm{M}$ calculates the position offset $\left(\Delta x_{M}, \Delta y_{M}, \Delta z_{M}\right)$ by using the position data and time obtained by the previous two times of localizations,

$$
\left\{\begin{aligned}
\Delta x_{M} & =\frac{x_{M}(n)-x_{M}(n-1)}{T(n)-T(n-1)} \\
\Delta y_{M} & =\frac{y_{M}(n)-y_{M}(n-1)}{T(n)-T(n-1)} \\
\Delta z_{M} & =\frac{z_{M}(n)-z_{M}(n-1)}{T(n)-T(n-1)}
\end{aligned}\right.
$$

where $x_{M}(n), y_{M}(n), z_{M}(n)$ are the coordinates of the $n$th localization, and $T(n)$ is the time of the $n$th localization, $n=1,2 \ldots$.

At the same time, node $\mathrm{M}$ sends a co-location request, the nodes $\mathrm{E}, \mathrm{F}, \mathrm{K}$ receive the request, calculate their position offsets $\left(\Delta x_{E}, \Delta y_{E}, \Delta z_{E}\right),\left(\Delta x_{F}, \Delta y_{F}, \Delta z_{F}\right),\left(\Delta x_{K}, \Delta y_{K}, \Delta z_{K}\right)$ and send them to node $\mathrm{M}$, node $\mathrm{M}$ calculates the distances $d_{E}, d_{F}, d_{K}$ from it to nodes $\mathrm{E}, \mathrm{F}$, and $\mathrm{K}$.

The underwater node movement has consistency in a short time [41], and its current position offset $(\Delta x, \Delta y, \Delta z)$ can be derived by using its last position offset and the neighbor nodes' position offsets,

$$
\left\{\begin{array}{l}
\Delta x=w_{M} \Delta x_{M}+w\left(d_{E}\right) \Delta x_{E}+w\left(d_{F}\right) \Delta x_{F}+w\left(d_{K}\right) \Delta x_{K} \\
\Delta y=w_{M} \Delta y_{M}+w\left(d_{E}\right) \Delta y_{E}+w\left(d_{F}\right) \Delta y_{F}+w\left(d_{K}\right) \Delta y_{K} \\
\Delta z=w_{M} \Delta z_{M}+w\left(d_{E}\right) \Delta z_{E}+w\left(d_{F}\right) \Delta z_{F}+w\left(d_{K}\right) \Delta z_{K}
\end{array}\right.
$$

where $W$ is a weight that inversely proportional to the distance between the nodes, $w_{M}+w\left(d_{E}\right)+w\left(d_{F}\right)+w\left(d_{K}\right)=1$.

Let $w\left(d_{E}\right)=\frac{L}{d_{E}}, \quad w\left(d_{F}\right)=\frac{L}{d_{F}}, w\left(d_{K}\right)=\frac{L}{d_{K}}$, we could get, 


$$
w_{M}+\left(\frac{1}{d_{E}}+\frac{1}{d_{F}}+\frac{1}{d_{K}}\right) L=1
$$

$w_{M}$ can be adjusted according to the actual situation, substitutes it to Eq. (10) to solve L, we can get $w\left(d_{E}\right), w\left(d_{F}\right), w\left(d_{K}\right)$.

According to the number of beacons could communicate to the node, the above formula can be written as

among them, $w+\sum w\left(d_{i}\right)=1$.

$$
\left\{\begin{array}{l}
\Delta \mathrm{x}=\mathrm{w}_{\mathrm{M}} \Delta \mathrm{x}_{\mathrm{M}}+\sum \mathrm{w}\left(\mathrm{d}_{\mathrm{i}}\right) \Delta \mathrm{x}_{\mathrm{i}} \\
\Delta \mathrm{y}=\mathrm{w}_{\mathrm{M}} \Delta \mathrm{y}_{\mathrm{M}}+\sum \mathrm{w}\left(\mathrm{d}_{\mathrm{i}}\right) \Delta \mathrm{y}_{\mathrm{i}} \\
\Delta \mathrm{z}=\mathrm{w}_{\mathrm{M}} \Delta \mathrm{z}_{\mathrm{M}}+\sum \mathrm{w}\left(\mathrm{d}_{\mathrm{i}}\right) \Delta \mathrm{z}_{\mathrm{i}}
\end{array}\right.
$$

Node $M$ can calculate its current position based on the position offset $\left(x_{M}, y_{M}, z_{M}\right)$,

where $T$ is the current localization time.

$$
\left\{\begin{array}{l}
x_{M}=[T-T(n)] \Delta x+x_{M}(n) \\
y_{M}=[T-T(n)] \Delta y+y_{M}(n) \\
z_{M}=[T-T(n)] \Delta z+z_{M}(n)
\end{array}\right.
$$

When the node is able to communicate with sufficient number of beacons, it exits the ICL and uses RCL again.

\section{Simulations}

In this section, we did experiments to analyze RCL and ICL algorithm. Based on the actual environment in the ocean, we use the MATLAB2016a platform to simulate the deployment and movement of nodes in the sea. The time for the node to send information is $0.1 \mathrm{~s}$, the seawater temperature and salinity are set to $15^{\circ} \mathrm{C}$ and $35 \%$ respectively, and the communication radius is $150 \mathrm{~m}$. The propagation speed of underwater acoustic waves is listed according to the underwater channel:

$$
\mathrm{c}=1449.30+4.6 \mathrm{~T}-0.055 \mathrm{~T}^{2}+0.00029 \mathrm{~T}^{3}+(1.34-0.01 \mathrm{~T}) \times(\mathrm{S}-35)+0.16 \mathrm{P}
$$

Where: $T$ represents the temperature of the seawater where the node is located, $S$ represents the salinity of the seawater where the node is located, and $P$ represents the pressure at the depth of the node.

First, the position of the node is calculated by Multilateration algorithm. Then the position obtained by Multilateration algorithm is corrected by RCL algorithm. Second, the results before and after the correction are compared with TP-TSFLA algorithm and SLA algorithm. At the same time, the performance of ICL algorithm in the case of insufficient beacons is analyzed and compared with Mobile Nodes Localization Algorithm (MNLS) [42].

The TP-TSFLA [14] and SLA [15] algorithms were chosen because they have many similarities with the RCL algorithm. The TP-TSFLA algorithm has two phases. The first phase is based on the particle swarm optimization algorithm to obtain the coordinates of the unknown nodes. The second stage is based on the distance-independent positioning algorithm of the circle to locate the unknown nodes left in the first stage. The positioning process of the RCL algorithm also requires two stages, and the geometric relationship between the node 
positions is used for positioning. The SLA algorithm first calculates the position of the unknown node in the overlapping area of the three beacon nodes, and then uses the iterative algorithm to extend the intersection area to the surrounding area to calculate the coordinates of other unknown nodes. The SLA algorithm has great similarity with the RCL algorithm proposed in this paper. It calculates the coordinates of the unknown node by calculating the intersection area of the beacon node. Through the experiment results, the performance and superiority of the RCL algorithm are analyzed.

The MNLS [42] algorithm is compared with the ICL algorithm because they are both mobile node-based positioning algorithm. The MNLS algorithm first predicts the motion trajectory of the node, and then performs ranging and positioning. ICL algorithm combines the inertial navigation position data with the motion information of the neighbor nodes to obtain the node position. They have great similarities and the comparison between them proves the superiority of our proposed ICL algorithm.

\subsection{RCL Simulation}

We use simulation software to simulate the distribution state of nodes in the ocean. Four beacon nodes and ten un-located nodes are randomly deployed in the region of $100 \mathrm{~m} * 100 \mathrm{~m} * 30 \mathrm{~m}$. The distance between the beacon and the un-located node is obtained by TDOA. The time interval for transmitting information is $0.1 \mathrm{~s}$, the seawater temperature and salinity are set to $15^{\circ} \mathrm{C}$ and $35 \%$, respectively, and the communication radius is $150 \mathrm{~m}$.

We first use the Multilateration algorithm to calculate the coordinates of 10 un-located nodes. Then the RCL algorithm is used to correct the obtained result, and compared with the error of Multilateration algorithm, as shown in Fig. 5. Then we use the RCL algorithm to compare with TP-TSFLA algorithm and SLA algorithm respectively, as shown in Fig. 6.

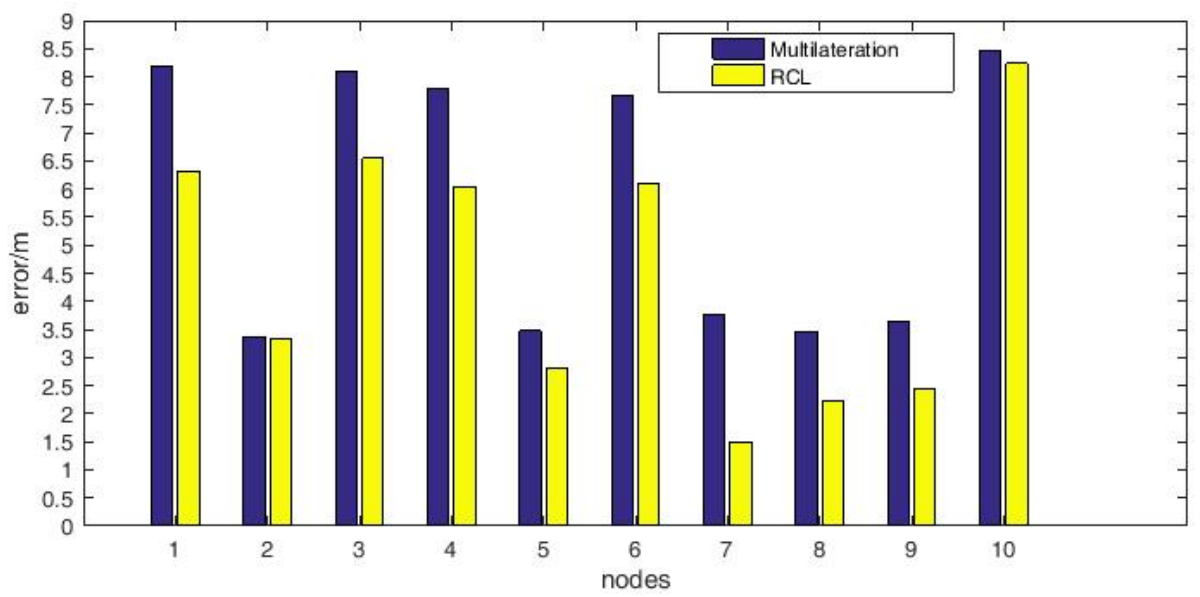

Fig. 5. Errors comparison of RCL and Multilateration

It can be seen from the error comparison results of RCL algorithm and Multilateration algorithm, as shown in Fig. 5, the maximum error and the average error of RCL algorithm are much smaller than those obtained directly by the Multilateration algorithm. This is because the error of the Multilateration algorithm is mainly derived from the ranging error, and the calculated rough position will be located on the line where the beacon node and the actual position are located, as shown in Fig. 2. The RCL algorithm makes the coarse position on this line closer to the precise position through the geometric relationship between the node 
positions, improving the position accuracy.

In addition, we can see that the errors of nodes 2, 5, 7, 8, 9 are smaller than other five nodes. This is because the error of the Multilateration algorithm is mainly influenced by the ranging error. The distance from the four beacons is different, and the ranging error is different. When using RCL, if the error calculated by the original algorithm is small, then the correction will be closer to the exact position.

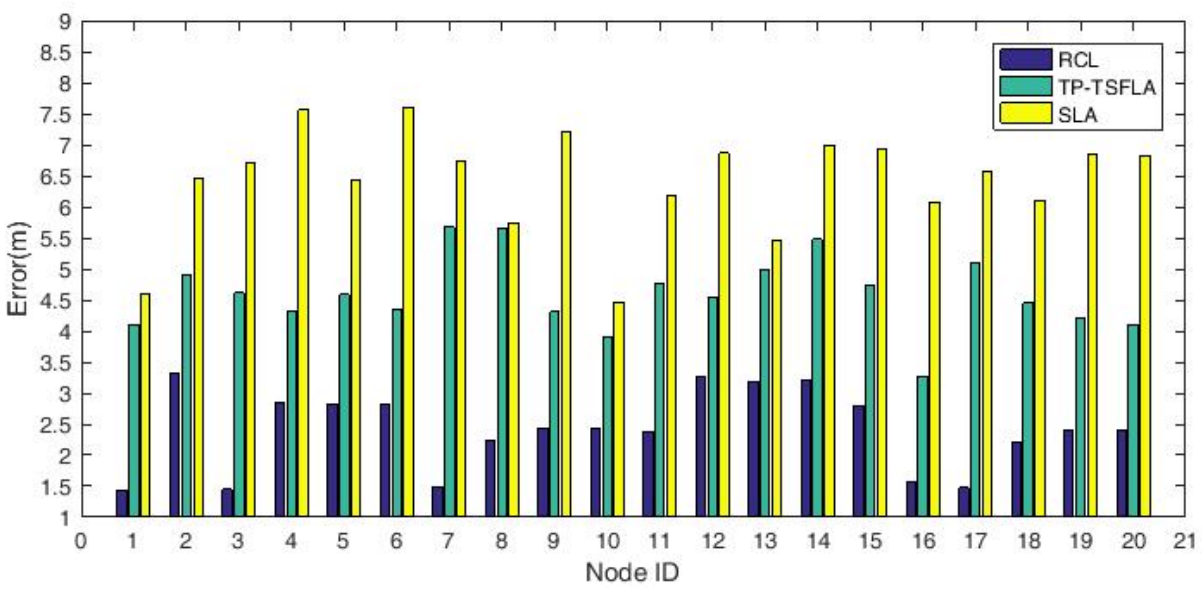

Fig. 6. Localization errors of RCL, TP-TSFLA and SLA

Fig. 6 shows the comparison results of the localization errors of RCL algorithm, TP-TSFLA algorithm and SLA algorithm. The comparison results between RCL algorithm and the two other algorithms can be seen in the figure. The average error of the TP-TSFLA algorithm is $4.583 \mathrm{~m}$, the average error of the SLA algorithm is $6.436 \mathrm{~m}$, and the average error of the RCL algorithm is $2.618 \mathrm{~m}$. The error of RCL algorithm is much smaller than TP-TSFLA algorithm and the SLA algorithm. This is because the errors of TP-TSFLA algorithm and SLA algorithm are derived from the ranging error. However, the RCL algorithm corrects the ranging error by the geometric relationship between the nodes, so its accuracy is greatly improved.

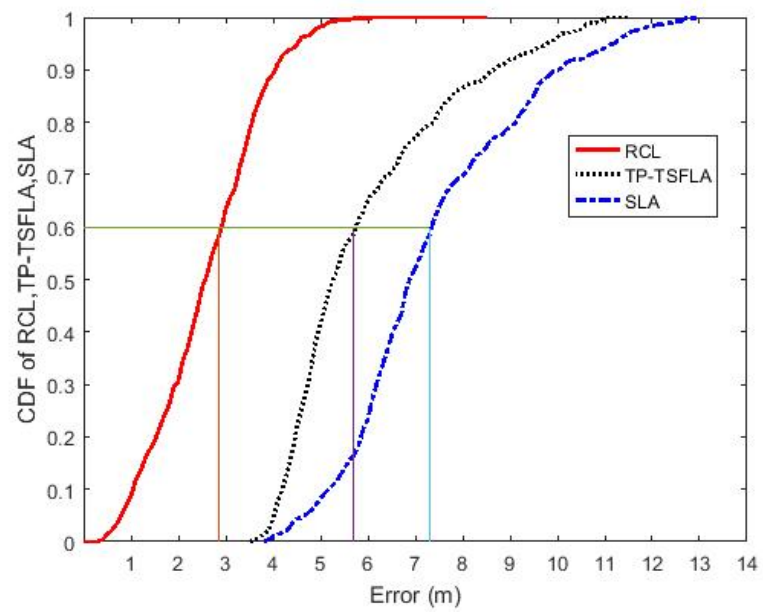

Fig. 7. CDF of localization errors 
In order to detect the performance of the RCL algorithm in the case of high-density deployment, we made the localization errors of RCL, TP-TSFLA, and SLA algorithms into CDF graphs. The CDF graphs can visually represent the distribution of errors in a particular area. We randomly deploy 500 nodes in the region of $100 \mathrm{~m} * 100 \mathrm{~m} * 30 \mathrm{~m}$, the depth of these nodes is between 0 and 30 meters from the sea surface. Fig. 7 shows the CDF of the localization errors of RCL algorithm, TP-TSFLA algorithm and SLA algorithm in the same ocean environment. As can be seen from the figure, the localization errors of RCL algorithm are significantly smaller than that of TP-TSFLA algorithm and SLA algorithm. When the CDF is 0.6 , the localization error of the RCL algorithm is between $2.5 \mathrm{~m}$ and $3 \mathrm{~m}$. However, the localization error of TP-TSFLA algorithm is greater than $5.5 \mathrm{~m}$, and the localization error of SLA algorithm is greater than $7 \mathrm{~m}$. Obviously, in the same ocean environment, the localization error of RCL algorithm is smaller. The above analysis fully proves the superiority of the RCL algorithm.

\subsection{ICL Simulation}

The ICL algorithm is mainly used when the node deviates from the deployment area, resulting in the inability to communicate with enough beacons in a certain period of time. We still randomly generate 10 unknown nodes and 4 beacon nodes, and then use the RCL algorithm mentioned above to calculate the first two position offsets of nodes, and then calculate the position of unknown nodes according to the ICL algorithm. We set the water flow direction to an angle of 45 degrees with the xy axis, the node moves 30 meters every 10 seconds, and the localization time interval is also 10 seconds. The specific process is shown in Fig. 8.

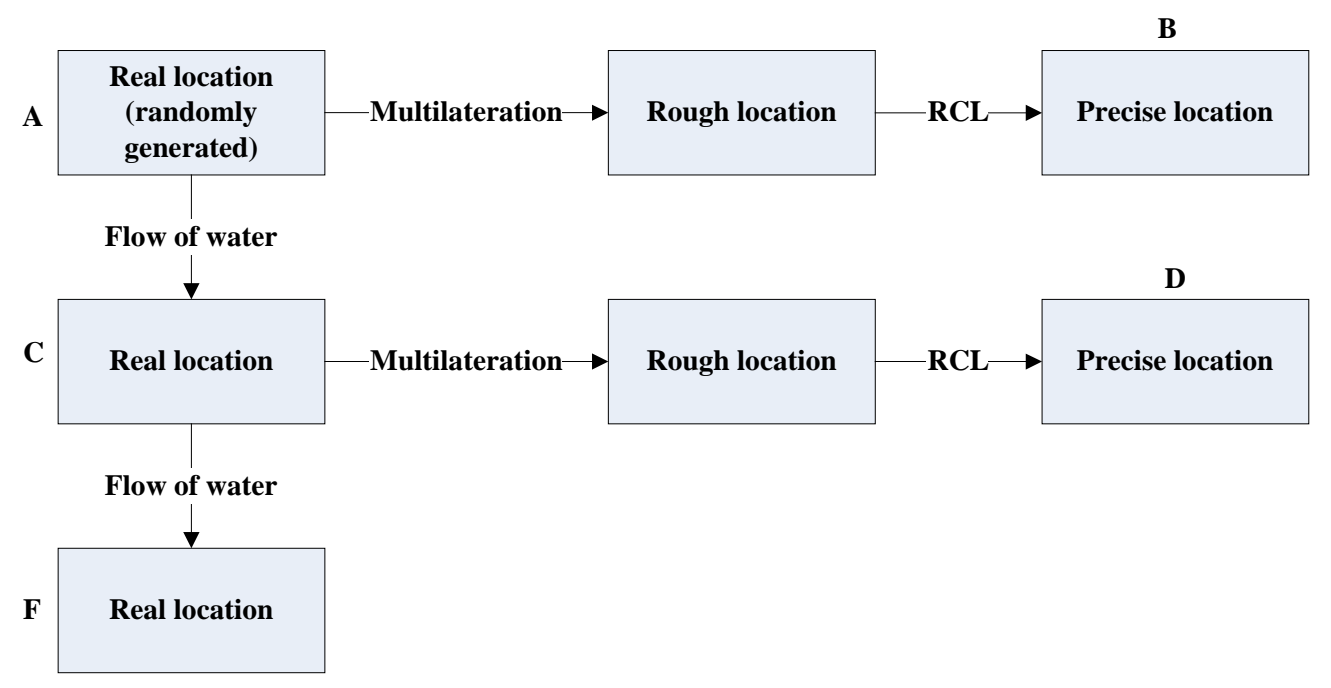

Fig. 8. Inertial coordination localization flowchart

We randomly generate the position $\mathrm{A}$ of the node, and then calculate the position $\mathrm{B}$ according to RCL algorithm. And then we use software to simulate the flow of water to get the position C. Next, we calculate the position D using RCL algorithm. The position F is obtained by simulating the flow of water according to the position C. From the position B and D, we can get the position offset of the node. From position $\mathrm{C}$ we can get the distance from the un-located nodes to the neighbor nodes. Using Eq. (11) and (12) we can calculate the position of the un-located node. We compare the calculated position with the position F to obtain a set of 
errors, and compare them with the errors of MNLS algorithm as shown in Fig. 9.

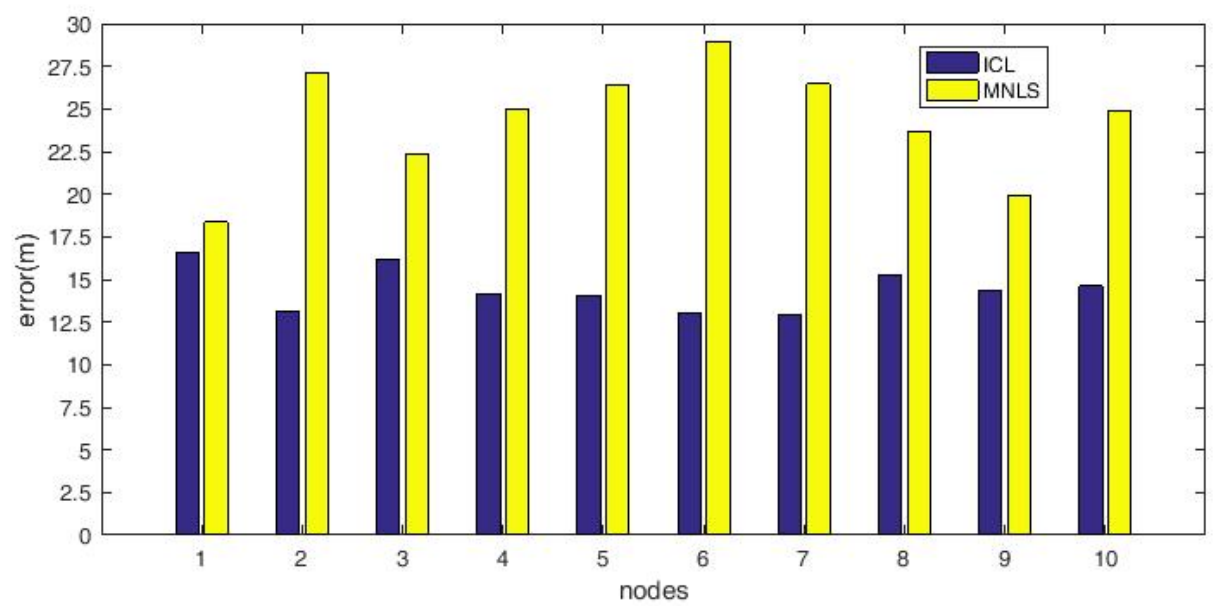

Fig. 9. Errors comparison of ICL and MNLS

It can be seen from the error comparison results in Fig 9. The maximum error and average error of ICL algorithm are much smaller than the error obtained by MNLS algorithm.

\subsection{Impacts of Nodes' Velocity on Localization Results}

In this section, we analyze the impacts of nodes' velocity on localization results. Keeping the original parameter settings, setting 10 unknown nodes and 4 known nodes, the water flow direction is 45 degrees from the $\mathrm{XY}$ axis, and the localization time interval is 10 seconds. We change the nodes' velocity, which are $3 \mathrm{~m} / \mathrm{s}, 5 \mathrm{~m} / \mathrm{s}$ and $7 \mathrm{~m} / \mathrm{s}$ respectively. The result is shown in Fig. 10.

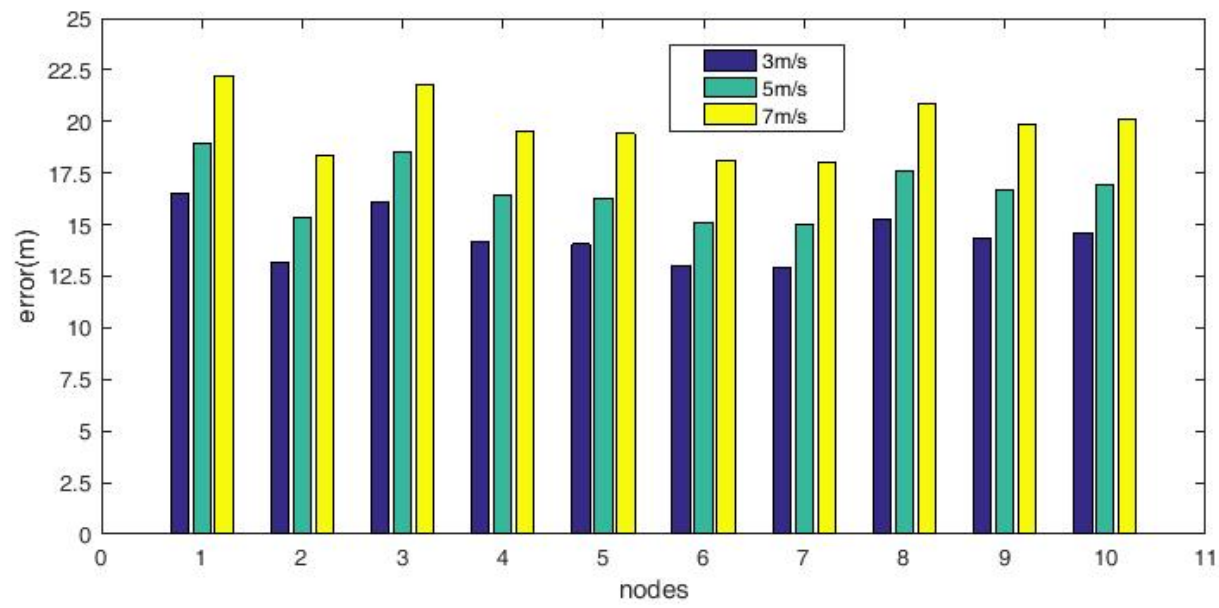

Fig. 10. Effect of the moving speed of the nodes 
It can be seen from Fig. 10 that the nodes' velocities have a certain influence on the error. When the nodes move faster, the errors are greater. But overall, the inertial coordination localization algorithm has relatively small errors.

From simulations, we can see that RCL and ICL algorithm for underwater acoustic sensor networks can reduce the ranging error and improve the position accuracy.

\section{Conclusion}

In this paper, we propose RCL and ICL algorithm to locate underwater sensor nodes. RCL algorithm corrects the ranging error by the geometric relationship between the node positions, and calculates the exact position of the node. It greatly reduces the cost of investment and the impact of other external environments. ICL algorithm obtains the node position by combined the inertial navigation with the motion information of neighbor nodes to improve positioning accuracy. Compared with TP-TSFLA algorithm, SLA algorithm and MNLS algorithm, RCL and ICL algorithm have significantly higher position accuracy under the same underwater environment, and have good practicability in the underwater wireless sensor network applications. In the future, we will continue to reduce the error rate of sensor network localization based on mobile restricted beacons, expand the applicability of the algorithm to different environments, and do more works on the multidimensional evaluation along different parameters, so that it can be better applied to different kind of underwater networks.

\section{References}

[1] Tran-Quang, Vinh, Thu Ngo-Quynh, and Minho Jo, "A Lateration-localizing algorithm for energy-efficient target tracking in wireless sensor networks," Ad Hoc \& Sensor Wireless Networks, 34, 1-4, 191-220, 2016.

[2] Rahmati, Mehdi, Seth Karten, and Dario Pompili, "SLAM-based underwater adaptive sampling using autonomous vehicles," in Proc. of OCEANS 2018 MTS/IEEE Charleston. IEEE, 2018. Article (CrossRef Link).

[3] Fadel E, Gungor V C, Nassef L, et al, "A survey on wireless sensor networks for smart grid," Computer Communications, 71(C), 22-33, 2015. Article (CrossRef Link).

[4] Liu S, Ozay M, Okatani T, et al, "Detection and pose estimation for short-range vision-based underwater docking,” IEEE Access, 7, 2720-2749, 2019. Article (CrossRef Link).

[5] Tsai, Pei-Hsuan, Rong-Guei Tsai, and Shiuan-Shiang Wang, "Hybrid localization approach for underwater sensor networks,” Journal of Sensors, vol. 2017, 2017. Article (CrossRef Link).

[6] Shon, Minhan, Minho Jo, and Hyunseung Choo, "An interactive cluster-based MDS localization scheme for multimedia information in wireless sensor networks," Computer communications, 35(15), 1921-1929, 2012. Article (CrossRef Link).

[7] Liu M, Guo X, Zhang S, "Localization based on best spatial correlation distance mobility prediction for underwater wireless sensor networks," in Proc. of Control Conference. IEEE, 7827-7832, 2015.

[8] Luo, Junhai, et al, "Research on localization algorithms based on acoustic communication for underwater sensor networks," Sensors, 18(1), 67, 2018. Article (CrossRef Link).

[9] Li Z, Guo Z, Hong F, et al., " $E^{2}$ DTS: An energy efficiency distributed time synchronization algorithm for underwater acoustic mobile sensor networks," Ad Hoc Networks, 11(4), 1372-1380, 2013. Article (CrossRef Link).

[10] Chen, Zhaohui, et al., "ULES: Underwater localization evaluation scheme under beacon node drift scenes," IEEE Access, 6, 70615-70624, 2018. Article (CrossRef Link).

[11] Han, Guangjie, et al., "Localization algorithms in large-scale underwater acoustic sensor networks: A quantitative comparison," International Journal of Distributed Sensor Networks, 
10(3), 379-382, 2014. Article (CrossRef Link).

[12] Sun D Y, Qian Z H, Han M F, et al, "Improved clustering analysis algorithm for multilateral location in wireless sensor networks," Chinese Journal of Electronics, 42(8), 1601-1607, 2014. Article (CrossRef Link).

[13] Jiang, Jian, Gang Wang, and K. C. Ho, "Sensor network based rigid body localization via semidefinite relaxation using arrival time and doppler measurements," IEEE Transactions on Wireless Communications, 18(2), 1011-1025, 2019. Article (CrossRef Link).

[14] Luo J, Fan L, "A two-phase time synchronization-free localization algorithm for underwater sensor networks,” Sensors, 17(4), 726, 2017. Article (CrossRef Link).

[15] Chang H Y, Kang H L, Moon H P, et al., "Sensor localization algorithms in underwater wireless sensor networks,” in Proc. of Iccas-Sice. IEEE, 1760-1764, 2009.

[16] Zoghi M, Kahaei M H, "Adaptive sensor selection in wireless sensor networks for target tracking,” Iet Signal Processing, 4(5),530-536, 2010. Article (CrossRef Link).

[17] Zhang S, Wang J, Liu X, et al, "Range-free selective multilateration for anisotropic wireless sensor networks," in Proc. of Mesh and Ad Hoc Communications and Networks. IEEE, 299-307, 2012. Article (CrossRef Link).

[18] Ying, Zhang, et al., "A localization method for underwater wireless sensor networks based on mobility prediction and particle swarm optimization algorithms,” Sensors, 16(2), 212, 2016. Article (CrossRef Link).

[19] Hari, Sai Krishna Kanth, et al., "Estimation of location and orientation for underwater vehicles from range measurements," 2018.

[20] Misra S, Ojha T, Mondal A, "Game-theoretic topology control for opportunistic localization in sparse underwater sensor networks," in Proc. of Mobile Computing IEEE Transactions on, 14(5), 990-1003, 2015. Article (CrossRef Link).

[21] Guo Ying, and Yutao Liu, "Localization for anchor-free underwater sensor networks," Computers \& Electrical Engineering, 39(6), 1812-1821, 2013. Article (CrossRef Link).

[22] V Mahajan, H Singh, "A review on underwater wireless sensor networks," International Journal of Advanced Research in Computer Science and Software Engineering, 5(5), 1576-1583, 2015.

[23] Maqsood H, Javaid N, Yahya A, et al., "Mobil-AUV: AUV-aided localization scheme for underwater wireless sensor networks," in Proc. of International Conference on Innovative Mobile and Internet Services in Ubiquitous Computing. IEEE, 170-175, 2016. Article (CrossRef Link).

[24] Kim S, Yoo Y, "High-precision and practical localization using seawater movement pattern and filters in underwater wireless networks," in Proc. of International Conference on Computational Science and Engineering. IEEE, 374-381, 2014. Article (CrossRef Link).

[25] Luo, Junhai, and Liying Fan, "A two-phase time synchronization-free localization algorithm for underwater sensor networks," Sensors, 17(4), 726, 2017. Article (CrossRef Link).

[26] Shit R C , Sharma S , Puthal D, et al, "Location of Things (LoT): A review and taxonomy of sensors localization in IoT infrastructure,” IEEE Communications Surveys \& Tutorials, 2018.

[27] Crasta N, Moreno-Salinas D, Pascoal A M, et al, "Multiple autonomous surface vehicle motion planning for cooperative range-based underwater target localization," Annual Reviews in Control, 46, 326-342, 2018. Article (CrossRef Link).

[28] Jamalabdollahi M, Zekavat S, "Time of arrival estimation in wireless sensor networks via OFDMA," in Proc. of Vehicular Technology Conference. IEEE, 1-5, 2015. Article (CrossRef Link).

[29] Wang Y, Ho K C, "TDOA source localization in the presence of synchronization clock bias and sensor position errors,” IEEE Transactions on Signal Processing, 61(18), 4532-4544, 2013. Article (CrossRef Link).

[30] Cui W, Wu S C, Wang Y Z, "A gossip-based AOA distributed localization algorithm for wireless sensor networks," Applied Mechanics \& Materials, 577, 841-846, 2014. Article (CrossRef Link).

[31] Ren M M, Xie Z J, Jin G, et al., "DV-Hop localization algorithm based on multi-mobile beacons," Computer Engineering, 40(10), 92-97, 2014. 
[32] Cheng X, Shu H, Liang Q, et al, "Silent positioning in underwater acoustic sensor networks," IEEE Transactions on Vehicular Technology, 57(3),1756-1766, 2008. Article (CrossRef Link).

[33] Erol M, Vieira L F M, Gerla M, "Localization with Dive'N'Rise (DNR) beacons for underwater acoustic sensor networks," in Proc. of The Workshop on Underwater Networks. DBLP, 97-100, 2007. Article (CrossRef Link).

[34] Erol M, Vieira L F M, Caruso A, et al, "Multi stage underwater sensor localization using mobile beacons," in Proc. of Second International Conference on Sensor Technologies and Applications. IEEE Computer Society, 710-714, 2008. Article (CrossRef Link).

[35] Erol M, Vieira L F M, Gerla M, "AUV-aided localization for underwater sensor networks," in Proc. of International Conference on Wireless Algorithms, Systems and Applications. IEEE, 44-54, 2007. Article (CrossRef Link).

[36] Isik M T, Akan O B, "A three dimensional localization algorithm for underwater acoustic sensor networks,” IEEE Press, 8(9), 4457-4463, 2009. Article (CrossRef Link).

[37] Ma Z, Liu Y, Shen B, "Distributed wireless sensor network localization algorithm MDS-MAP(D),” Journal of Communications, 29(6), 57-62, 2008.

[38] Jung-Sup Um, “Location sensors,” Drones as Cyber-Physical Systems, 143-176, 2019. Article (CrossRef Link).

[39] Yan Y, Wang H, Xuan W, "A novel least-square method of source localization based on acoustic energy measurements for UWSN," in Proc. of IEEE International Conference on Signal Processing, Communications and Computing. IEEE, 1-5, 2011.

[40] Guo Z W, Luo H J, Hong F, et al, "Research progress of underwater wireless sensor networks," Computer Research and Development, 47(3), 377-389, 2010.

[41] Caruso A, Paparella F, Vieira L F M, et al, "The meandering current mobility model and its impact on underwater mobile sensor networks," in Proc. of INFOCOM 2008. The Conference on Computer Communications. IEEE, 221-225, 2008. Article (CrossRef Link).

[42] Wang M L, Song A J, "Positioning of 3D underwater sensor network based on mobile node," Computer System Application, 23(10), 162-166, 2014. 


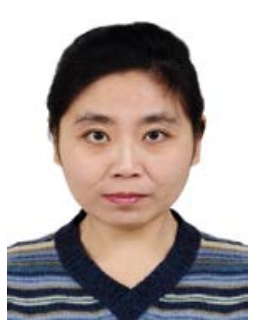

Ying Guo received her B.E. degree from Qingdao University of Science and Technology, in 2007. She received her Ph.D. degree in the Department of Computer Science and Technology from Ocean University of China, in 2010. She is an associate professor in Qingdao University of Science and Technology, and a visiting scholar in Arizona State University. Her research interests include wireless sensor networks, underwater acoustic networks and Internet of Things.

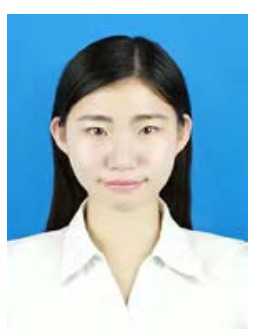

Xiaoyue Kang is currently pursuing her B.E. degree in computer science and technology at Qingdao University of Science and Technology. Her research interests include underwater acoustic sensor networks, Internet of Things.

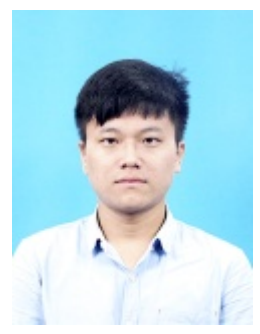

Qinghe Han is currently pursuing his B.E. degree in computer science and technology at Qingdao University of Science and Technology. His research interests include network localization, underwater sensor networks, and embedded systems.

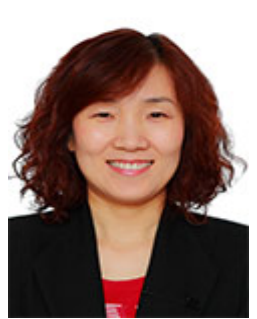

Jingjing Wang received her B.E. degree from Qingdao University of Science and Technology. She received her Ph.D. degree from Ocean University of China. She is a professor in Qingdao University of Science and Technology. Her research interests include wireless networks, sensor networks, underwater networks communication. 